\title{
Three-Dimensional Virtual-Reality Surgical Planning and Soft-Tissue Prediction for Orthognathic Surgery
}

\author{
James Xia, Member, IEEE, Horace H. S. Ip, Member, IEEE, Nabil Samman, Helena T. F. Wong, Jaime Gateno, \\ Dongfeng Wang, Richie W. K. Yeung, Christy S. B. Kot, and Henk Tideman
}

\begin{abstract}
Complex maxillofacial malformations continue to present challenges in analysis and correction beyond modern technology. The purpose of this paper is to present a virtual-reality workbench for surgeons to perform virtual orthognathic surgical planning and soft-tissue prediction in three dimensions. A resulting surgical planning system, i.e., three-dimensional virtual-reality surgical-planning and soft-tissue prediction for orthognathic surgery, consists of four major stages: computed tomography (CT) data post-processing and reconstruction, three-dimensional (3-D) color facial soft-tissue model generation, virtual surgical planning and simulation, soft-tissue-change preoperative prediction. The surgical planning and simulation are based on a 3-D CT reconstructed bone model, whereas the soft-tissue prediction is based on color texture-mapped and individualized facial soft-tissue model. Our approach is able to provide a quantitative osteotomy-simulated bone model and prediction of postoperative appearance with photorealistic quality. The prediction appearance can be visualized from any arbitrary viewing point using a low-cost personal-computer-based system. This cost-effective solution can be easily adopted in any hospital for daily use.
\end{abstract}

Index Terms-Maxillofacial deformity, orthognathic surgery, soft-tissue-change prediction, three-dimensional surgical planning and simulation, virtual reality.

\section{INTRODUCTION}

I T IS frequently difficult to make a precise surgical plan to correct for complex maxillofacial deformity using conventional methods. Traditional surgical planning for orthognathic surgery is determined by cephalometric analysis, and clinical and aesthetic judgment.

Three-dimensional (3-D) studies in medicine started at the beginning of the 1970's [1]. The cross-sectional imaging capability of computed tomography (CT) [2] and 3-D reconstruction have led to a tremendous leap in diagnostic radiology. Although cross-sectional slices avoid superimposition of adjacent structures and permit high-resolution details of bone, 3-D rendered

Manuscript received March 28, 2000; revised October 3, 2000. This work was supported in part by the University of Hong Kong under Committee on Research and Conference grants.

J. Xia was with the Oral and Maxillofacial Surgery Department, The University of Hong Kong, Hong Kong. He is now with the Department of Oral and Maxillofacial Surgery, Dental Branch, University of Texas at Houston, Houston, TX 77030 USA.

H. H. S. Ip, H. T. F. Wong, and C. S. B. Kot are with the Department of Computer Science, City University of Hong Kong, Kowloon, Hong Kong.

N. Samman, D. Wang, R. W. K. Yeung, and H. Tideman are with the Oral and Maxillofacial Surgery Department, The University of Hong Kong, Hong Kong.

J. Gateno is with the Department of Oral and Maxillofacial Surgery, Dental

Branch, University of Texas at Houston, Houston, TX 77030 USA.

Publisher Item Identifier S 1089-7771(01)02028-3. visualization provides the radiologists and surgeons with readily recognizable images of complex anatomic structures. It can exactly record and represent the life size and shape of bone for precise bone surgery planning and simulation.

Orthognathic surgery attempts to reestablish functional and aesthetic anatomy by repositioning displaced bones or by grafting and recontouring the deformed bone contour. Softtissue changes usually accompany these skeletal alterations, but, despite the advance in imaging modalities, the prediction soft-tissue deformation upon the bone structure change still poses major challenges to both surgeons and computer scientists. Although there are several methods to capture the softtissue contours, such as laser scanning [3], Cyberwere [4], the Moiré imaging technique [5], 3-D digitizer [6], and 3-D photographic method [7], to quantify bone-soft-tissue relations and to predict soft-tissue changes remain as open research problems.

Surgical planning, postoperative prediction, and visualization requires a combination of the knowledge between medicine and computer graphics. With respect to the point presented earlier, the purpose of this paper is to present a virtual-reality environment for orthognathic surgical planning. The environment provides facilities for a surgeon to make surgical plans, to simulate various orthognathic surgical procedures as they are routinely performed in an operating room, and to predict soft-tissue changes before operation. Furthermore, we present a surgical-planning system, which is termed the three-dimensional virtual-reality surgical-planning and soft-tissue prediction for orthognathic surgery (3DVRSP). The development of the 3DVRSP system is based on our previous studies on 3-D color facial-model generation [8], 3-D virtual osteotomy [9], and 3-D soft-tissue planning and prediction [10].

\section{VRSP OVERVIEW}

The virtual-reality workbench 3DVRSP consists of four major functions:

1) CT data post-processing and reconstruction;

2) 3-D color facial soft-tissue model generation;

3) virtual surgical planning and simulation;

4) soft-tissue-change prediction before operation (Fig. 1).

Mensurations such as linear and spatial measurements are also included as supplemental functions. Anatomy modeling and visualization were based on 3-D reconstructed axial CT data. Surgical planning and osteotomy simulation were made on the reconstructed 3-D bone structures, whereas soft-tissue changes 


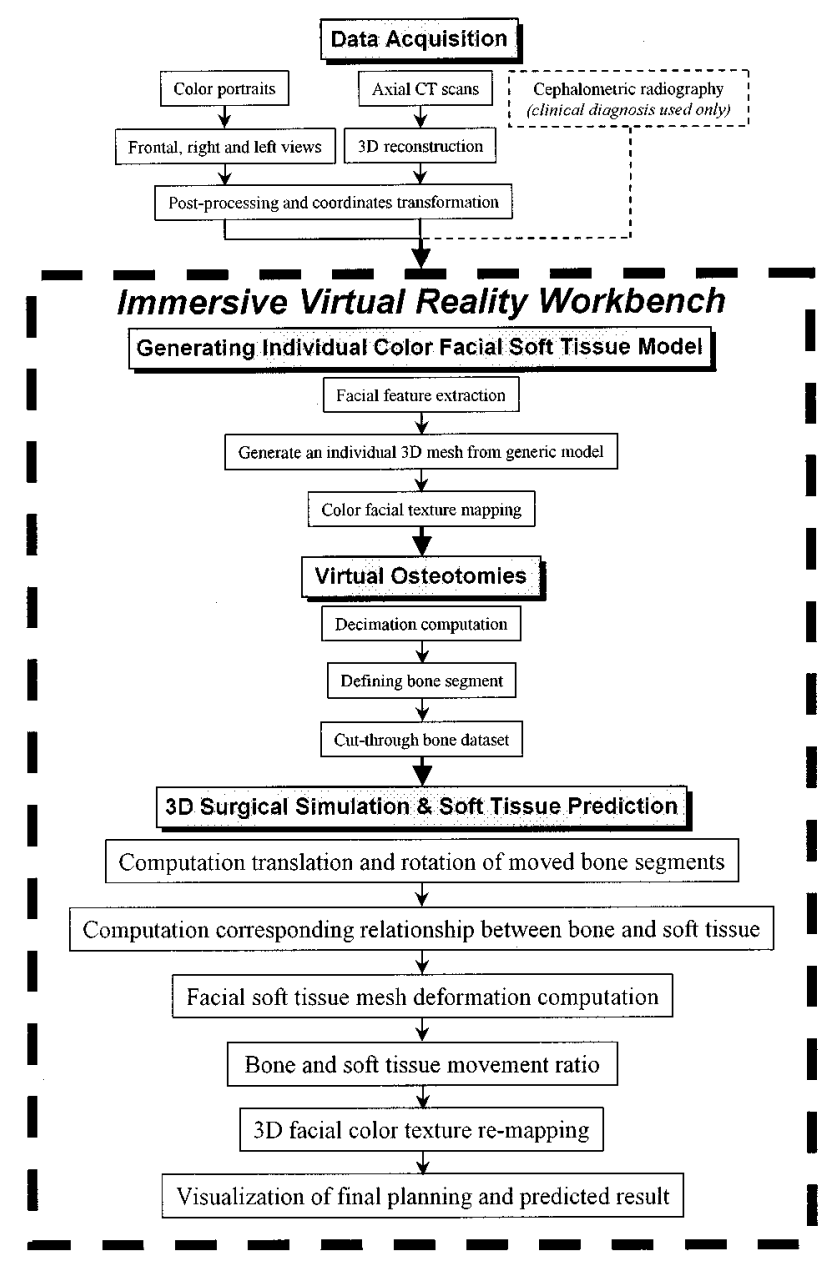

Fig. 1. System overview of 3DVRSP system.

were then predicted on the individualized and color texturemapped facial soft-tissue model.

More interestingly, the approach here allows an immersible virtual-reality surgical planning environment to be implemented on a relatively low-cost personal computer (PC)-based system when high-end graphics workstations were normally required. Within the virtual environment (or "Cyberspace") of VRSP, an surgeon can get hold of a virtual "scalpel" to operate on a "virtual" patient, to execute various surgical plans, and to visualize and predict the postoperative results through VRSP's 3-D virtual osteotomies and 3-D soft-tissue prediction facilities (Fig. 2). VRSP outputs orthognathic surgical plans and a 3-D color photorealistic patient's facial soft-tissue model. The osteotomy-simulated bone model and predicted color model can be rotated and visualized from any point-of-view.

An IBM-compatible PC was configured as an Intel Pentium-II 400 processor (Intel Inc., Santa Clara, CA) with 256-MB RAM and a Matrox Millennium G400 AGP display card (Matrox Graphics Inc., Québec, QC, Canada). An iomega ZIP (Iomega Corp., Roy, UT) drive is used to digitally transfer CT data from a CT scanner to our PC.

To create a virtual-reality workbench, the virtual-reality devices, such as stereo glasses and a 3-D flying mouse, are also applied for this study. The Logitech 3-D flying mouse system (Logitech Inc., Fremont, CA) is used as a virtual "scalpel."

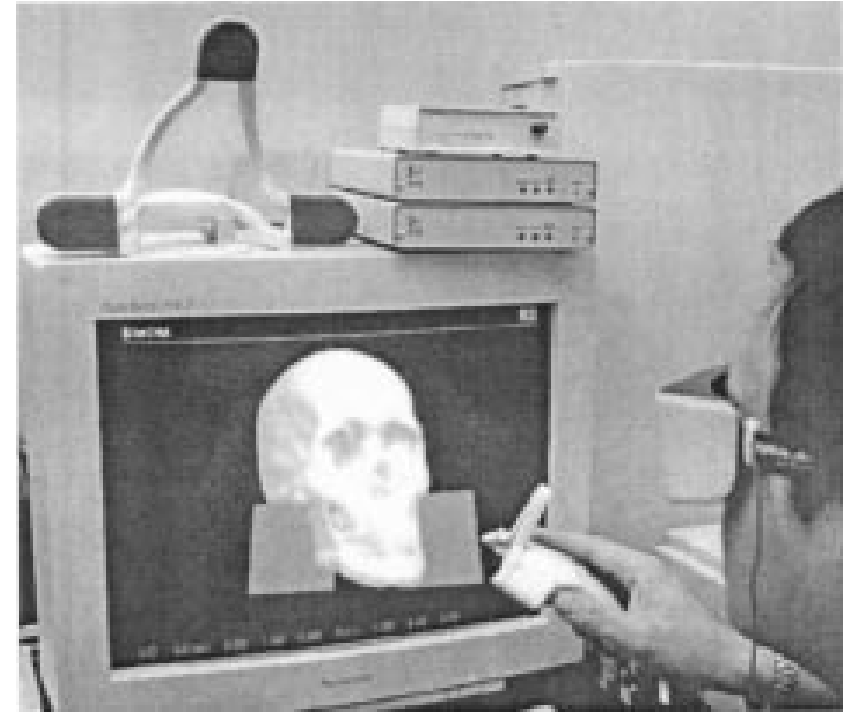

Fig. 2. Surgeon immersed into virtual-reality environment to operate on a virtual "patient."

CrystalEyes 2 eyewear (StereoGraphics Corporation, San Rafael, CA) is a device used to visualize stereoscopic images in Cyberspace.

The operating system was a Microsoft Windows NT Workstation 4.0 with Service Package 4, and all the programs were developed by Microsoft Visual $\mathrm{C}^{++} 6.0$ (Microsoft Corporation, Redmond, WA).

OpenGL (Silicon Graphics Inc., Mountain View, CA), the environment for developing portable and interactive 3-D graphics applications, was used for developing interactive two-dimensional (2-D) and 3-D visualization.

VTK (Kitware Inc., Clifton Park, NY), a visualization toolkit for developing computer graphics and visualization algorithms, was used for programming surface rendering and decimation computation.

\section{Clinical Data AcQuisition ANd ApPlication}

\section{A. Clinical Data Source}

Fourteen patients with maxillofacial deformities were randomly selected from a large pool of patients awaiting surgical correction of their deformity from December 1995 to December 1998. All patients were evaluated at the Surgical-Orthodontic Joint Assessment Clinic, Oral and Maxillofacial Surgery, University of Hong Kong.

Standard cephalometric radiographs were taken with a $165-\mathrm{cm}$ tube-to-film distance, $15-\mathrm{cm}$ patient midsagittal plane-to-film distance, and with a magnification ratio of $110 \%$. Diagnosis and preliminary operative plan were made by the cephalometric radiographs and clinical examination.

The patient's portraits were photographed as conventional medical documentation. CT scanning was carried out especially for this study. The rules of data acquisition are as follows [10].

1) The patient's facial expression is natural.

2) The teeth should be in occlusion.

3) View angles should be at $0^{\circ}, 90^{\circ}$, and $270^{\circ}$ during photography. 
4) The corresponding facial organ's position, i.e., the mouth, should be as similar as possible between CT scanning and photography.

5) The Frankfort Plane should be parallel to the horizontal plane as close as possible during photography, whereas the Frankfort Plane of the supine patient need to be vertical in relation to the horizontal plane during CT scanning.

6) Head and jaw's movements and facial expressions are prohibited during CT scanning.

7) Both portraits and CT scanning should be taken in the same period (normally within one week of each other).

\section{B. Color-Portrait Photography}

The frontal, right-, and left-side views of the patient's face in the natural head position were photographed separately by a Medical-Nikkor $120 \mathrm{~mm} / \mathrm{f} 4$ lens (Nikon Corporation, Tokyo, Japan). The magnification was fixed at $1: 8$ or $1: 11$, depending on the height of the patient's head. The central focal axis of the lens was adjusted to remain horizontal during the photography process. The patient's Frankfort Plane was parallel to the horizontal plane. Portraits were then scanned as 24-bit images into the computer via a Nikon LS-20 film scanner (Nikon Corporation, Tokyo, Japan) and the digitized images were resampled to $360 \times 550$ pixels each.

\section{CT Data Acquisition}

All patients were examined by a GE Pace CT Scanner (GE Medical Systems, Waukesha, WI) at the Hong Kong Adventist Hospital, Hong Kong, with a thickness of $2.0 \mathrm{~mm}$ and original $512 \times 512$ matrix with 16 bits. The display fields of view (FOV) ranged from 21 to $30 \mathrm{~cm}$. CT scanning was performed at $120 \mathrm{KV}, 80 \mathrm{~mA}$, and $2.0 \mathrm{~s}$ each by 99 125 slices, depending on the height of the patient's head. The Frankfort Plane of the supine patient was vertical in relation to the horizontal plane. A rubber band was used to restrict the patient head's movement during CT scanning. The 2-D axial slices started from the submandibular region and covered the whole head. All the raw data of CT scans were digitally transferred from the CT scanner to our PC by an iomega ZIP drive without losing any signal.

\section{METHODS}

\section{A. Processing of CT Data and 3-D Unique Coordinate System}

1) Processing of CT Data: For the true perspective of data visualization, image-processing techniques were used to manipulate image contents to improve the results of subsequent processing and interpretation. The post-processing functions included: 1) removing undesired objects; 2) masking individual bone structure; and 3) the bone-structure enhancement.

Segmentation was the process of classifying pixels in the image or volume [11]. Depending on the scene complexity, it can be one of the most difficult tasks in the visualization process. Interactive segmentation was used if the threshold filter was not able to mask the bone in the $\mathrm{CT}$ dataset during the marching cubes process.

2) 3-D Reconstruction: A volumetric dataset was then prepared by using the marching cubes algorithm [12], [13] from the

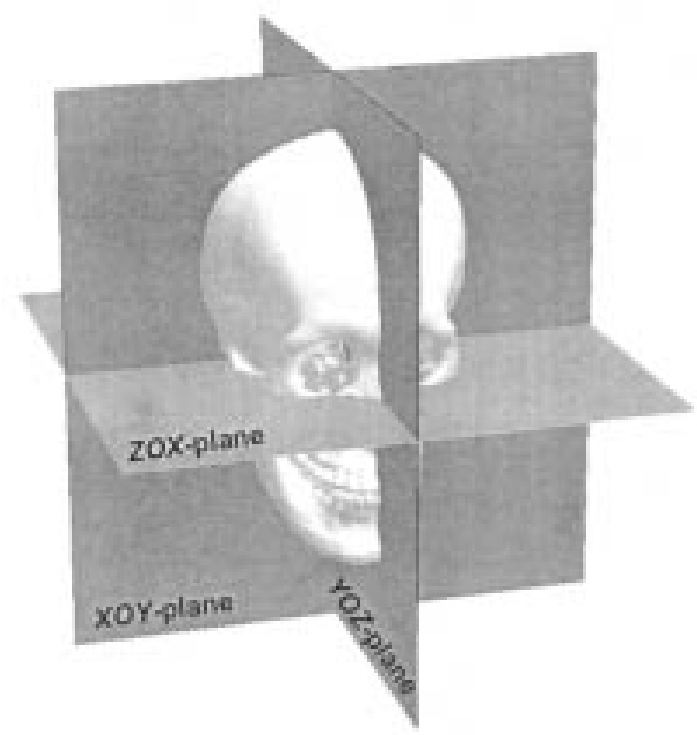

Fig. 3. 3-D coordinate system transformation.

processed CT raw data. For fast rendering and shading, all the succeeding operations were based on this volumetric dataset. The original CT slices were discarded in the subsequent processing.

3) Unique 3-D Coordinate System: The view coordinate system represented what was visible to the observer. The $x$ and $y$ coordinates specified location of object in a plane, whereas the $z$ coordinate represented the depth. The original coordinates in the CT dataset were transformed to the patient's coordinate system, accounting for slight malpositioning of the patient during the CT scanning [8]-[10].

A 3-D spatial coordinate system should be defined for visualization and further process. Five landmarks were needed to be interactively digitized on the reconstructed skull: nasion, rightand left-hand-side porion and right- and left-hand-side orbitale. These landmarks could usually be easily located on the frontal, right- and left-hand-side views of reconstructed bone visualization.

The horizontal plane was defined by right- and left-hand-side porion, and the average coordinates of the right and left orbitale. The frontal plane was perpendicular to the horizontal plane and through the right- and left-hand-side porion. The midsagittal plane was set perpendicular to the horizontal plane and the frontal plane, and through the nasion (Fig. 3).

After generating the 3-D coordinates, the polygons and triangles of the reconstructed models were transformed from the world coordinate system into the view coordinate system via vector product computing. Like cephalometric analysis, 3-D visualization and manipulation could be performed in this unified 3-D spatial coordinates repeatedly.

\section{B. 3-D Facial Soft-Tissue Model Generation}

Two separate techniques were applied during this stage of the process: individualized facial soft-tissue model generation from a generic mesh and color texture mapping.

1) Generic Facial Soft-Tissue Model: Our generic facial model is a triangular mesh consisted by 2665 vertices and 5302 


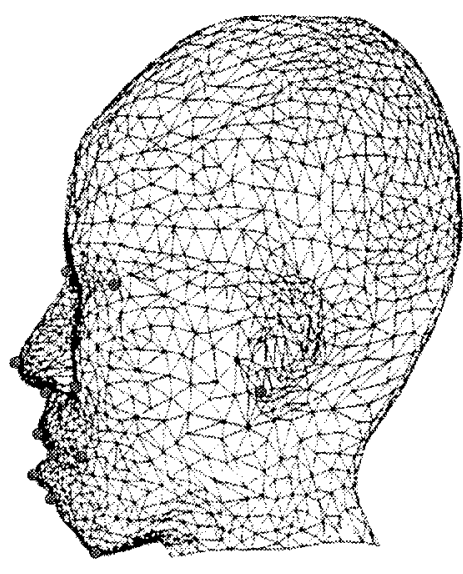

$270^{\circ}$ view

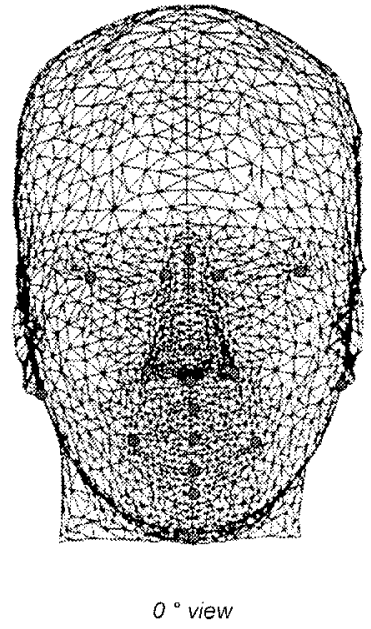

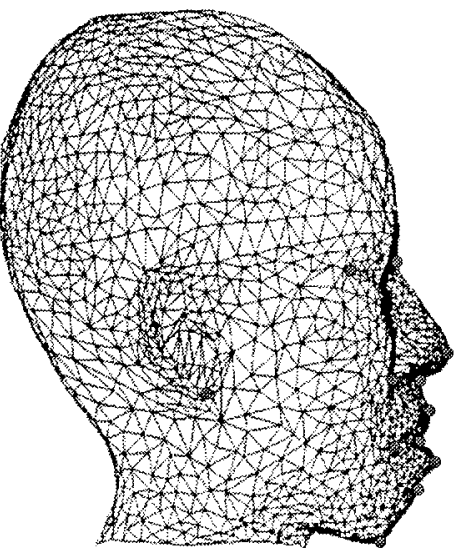

$90^{\circ}$ view

Fig. 4. Registration on the generic mesh.

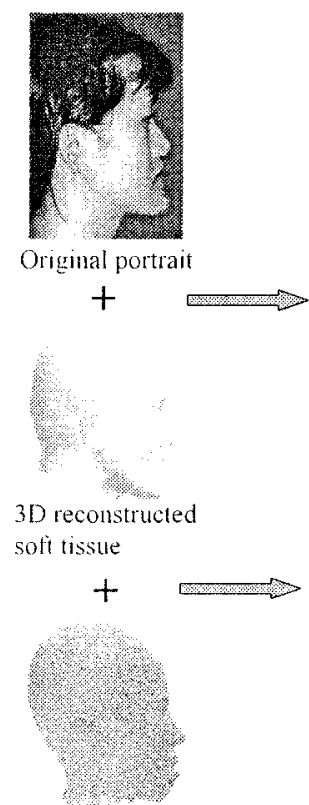

Generic mesh

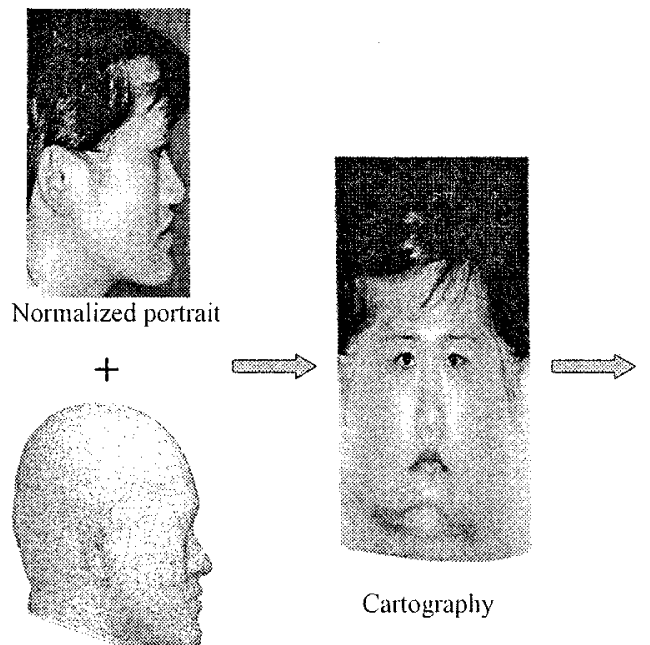

Individualized inesh

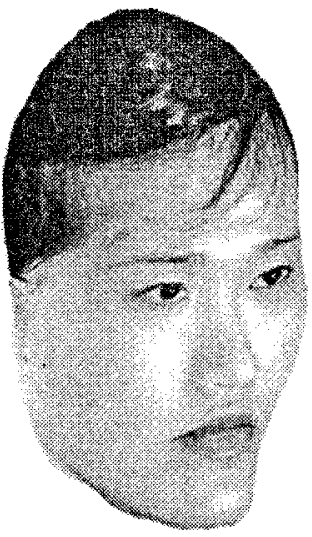

$3 D$ color facial model

Fig. 5. Generating 3-D individual color facial model.

triangles. This generic mesh was created from 3-D bone and soft tissue, which have been reconstructed from CT scans of a real human head. It contained the corresponding relationship of the coordinates between bone structure and soft tissue. Each coordinate on the bone surface had its own unique projection to the soft-tissue mesh. When each point on the bone surface was changed, the corresponding coordinate of soft tissue would be changed accordingly. This allows us to simulate soft-tissue movement as a result of bone movement. Additionally, there were a series of built-in vertices representing facial outlines and features.

2) 3-D Color Facial-Model Generation: Facial feature and outline key vertices were extracted from the 3-D reconstructed soft tissues from CT scans (Fig. 4), the generic mesh was then individualized as a texture coordinates by correspondence matching, interpolation, and transformation computation based on these fiducial points.

The texture mapping was a procedure similar to pasting a picture to the surface of an object. Three digitized color portraits with the "third" dimension from reconstructed soft tissue were blended into a cartograph as a texture map.

After the cartograph was texture mapped on to the 3-D head model, a photorealistic model of a human head was generated from frontal, right-, and left-hand-side real color portraits.

Individualized Facial-Model Generation from Generic Model: A soft-tissue color facial model, containing a bone-soft-tissue relationship and facial color information, was generated from a generic model and color portraits (Fig. 5).

The feature points characterized each facial organ and outline of the patient's face, with the same definition as the built-in 

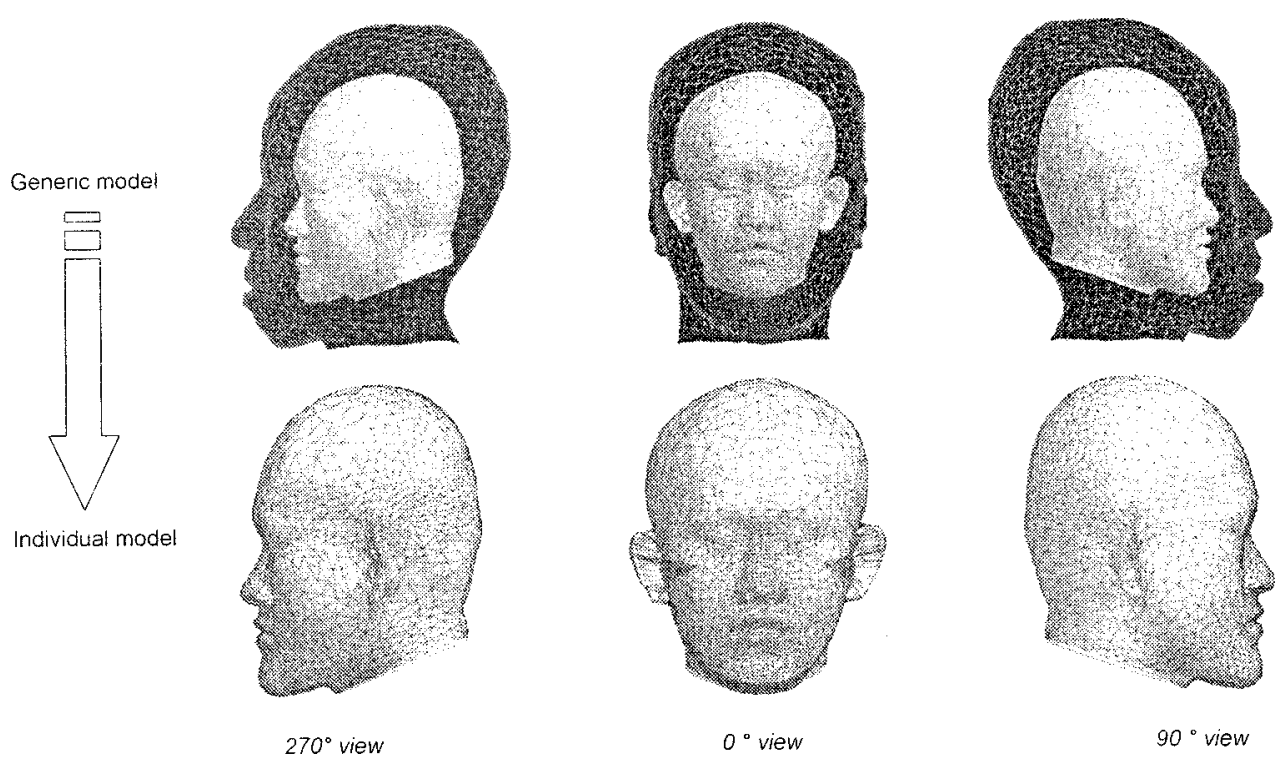

Fig. 6. Individualizing generic model.

landmarks in the generic mesh, and were registered interactively on both of the color portraits and 3-D reconstructed soft tissues.

To approximate an individualized facial mode, the facial outline and features of the organs should be mapped to the generic mesh first. The translation vectors were automatically computed by matching the feature vertices on the generic model to the corresponding feature points. A transformation vector field, which defined the translation vectors for both feature and nonfeature vertices in the generic model and transformed the generic model to individual for right-hand side, frontal and left-hand-side views, was also automatically estimated using the sets of translation vectors and bilinear interpolation. The outline and facial organs were correctly created although the nonfeature areas, such cheek area, might still be slightly deformed on the mesh.

To generating the fully individualized facial model, the vertices without feature landmarks should be accurately remapped onto the 3-D reconstructed images by an automatic and timeconsuming computation. Superimposing this approximated individualized mesh and reconstructed soft-tissue surface together by the facial outline and feature vertices, the nonfeature and mispositioned vertices on the mesh were displaced to the intersection points of the reconstructed surface along the ray projections from the $y$-axis. The individual mesh could then be precisely generated (Fig. 6). It contained all the 3-D information transformed from the 3-D reconstructed soft-tissue and bone-softtissue corresponding relationship from the generic mesh.

Color Texture Mapping: Although the facial soft-tissue model was precisely individualized, it was still lacking color information. Texture mapping was used to paste color onto this model.

Color texture mapping requires two elements: a texture map, where the texel could be obtained from, and a texture-coordinate where the texel could be pasted to. To define a texture map, a cartograph was generated from three digitized color portraits and reconstructed soft tissue. We assume each point on the car-

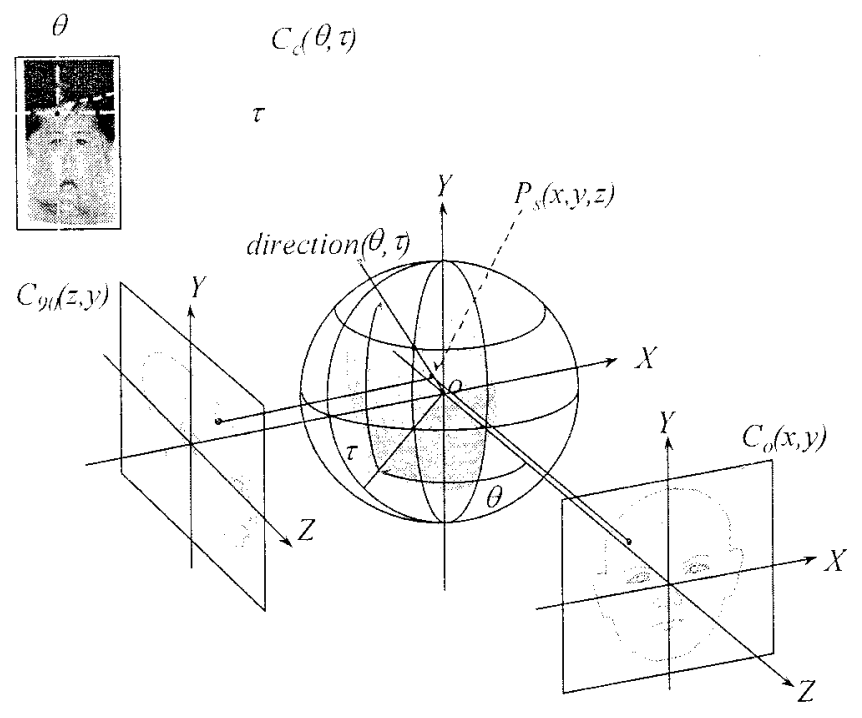

Fig. 7. 3-D facial texture mapping from cartography.

tograph, as corresponding to a sphere coordinate. Projecting a ray from the $y$-axis to a point, along this direction, an intersection point $P_{s}(x, y, z)$ could be found on the reconstructed CT soft-tissue surface. This point was projected on to the frontal view at $P_{0}(x, y)$ and lateral view at $P_{90}(z, y)$ of the portraits, respectively. Blending these three points together, the color information and coordinate of this pixel were generated as a cartographic pixel (Fig. 7). By repeating the above procedures, a complete cartograph with 3-D coordinates and full color information was generated as a texture map.

To generate a texture-mapped mesh projection in an arbitrary view direction, the texture coordinate on the cartograph for each triangle vertex on the mesh was calculated as the ideal relationship first. The color textures were then mapped onto the individualized facial model and the texture-mapped model could be visualized in real time. 


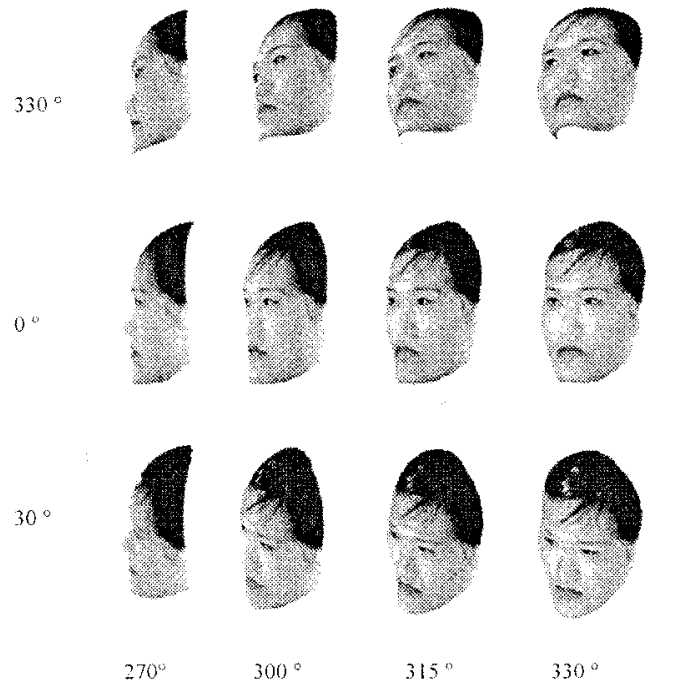

Fig. 8. Sequence of 3-D color facial model.
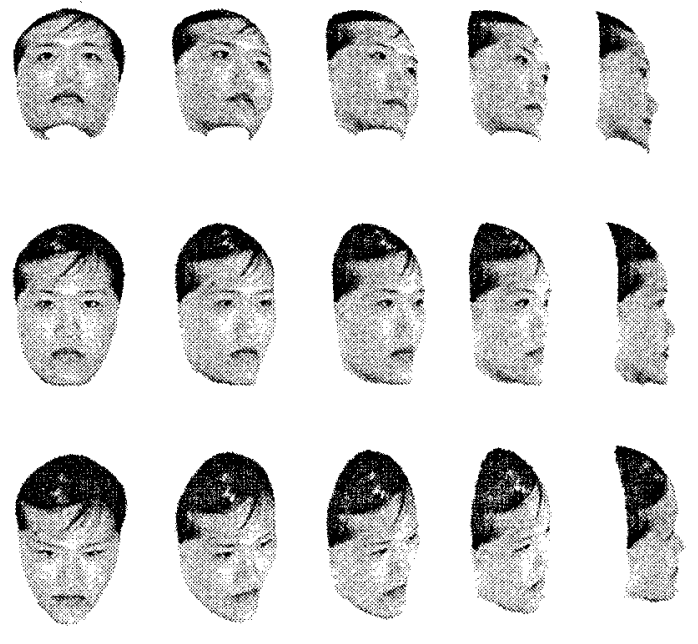

$0^{\circ}$

$45^{\circ}$

$60^{\circ}$

$90^{\circ}$

The patient might close his/her eyes during CT scanning, the eye features on the individualized model would also be represented as closed eyes. Instead of point-to-point mapping, the color information of eye details was extracted en bloc from 3-D color portraits (cartograph) and mapped onto the individualized model directly.

An individualized color texture-mapped facial model with photorealistic quality could be rotated freely and visualized from any viewpoint at real-time speed (Fig. 8). All the procedures described in the texture-mapping stage were fully automatic, no interactive operation was required, although it involved very complex computations.

\section{3-D Virtual Osteotomy Planning and Simulation}

This stage cut through the 3-D reconstructed bone dataset with a surface and then displayed interpolated data values on the surface in order to achieve the virtual osteotomy simulation.

The bone surface was represented by millions of polygons and triangles. A fully automatic decimation computation [14] was first applied on the reconstructed bone surface to reduce the triangles up to $70 \%$ in order to keep a balance of maintaining more bone structure details and obtaining an acceptable speed of rendering and a reasonable interval of feedback during cutthrough of the bone dataset.

The data cutting operation required two pieces of information: a definition for the surface (cutting surface) and a dataset to cut (which bone to be cut). It was assumed that the cutting surface was defined by an implicit function (virtual knife). Fig. 2 showed the cutting planes of bilateral subsigmoid osteotomies were defined by moving a 3-D mouse. A property of implicit functions was to convert a position into a scalar value. This property in combination with a contouring algorithm, i.e., marching cubes, to generate the cut surfaces.

Scalars were generated for each point of each cell of the CT dataset, and then contour the surface value $F(x, y, z)=$ 0 . For each cell, function values were generated by evaluation $F(x, y, z)$ for each cell point. If all the points evaluated either

positive or negative, then the surface did not cut the cell. However, if the points evaluated both positive and negative, then the surface passed through the cell. A contouring algorithm was then applied to generate the isosurface on the cutting surface.

The virtual osteotomy was performed interactively. The surgeon needed to decide which osteotomy would be used. The osteotomy was performed intraoperatively based on the surgeon's clinical experience. The immersible virtual-reality workbench simulated the environment of the operating room.

The decimated skull was subdivided into osteotomized segments with a corresponding overlying skin region. This was accomplished by visually editing the 3-D bone surface models with a virtual knife. The object (bone) was selected and separated into two parts immediately after defining the cutting plane (cutting through the bone dataset).

In the virtual osteotomy simulation, the binary tree structure was used to identify the bone segments during maxillary and mandibular osteotomies. Each bone segment was assigned a unique element identity (ID) automatically. A nonlinear structure, called a tree, consisted of nodes, branches, and leaves. In the simulation of the operative procedures of a segmentalized Le Fort I osteotomy and bilateral vertical subsigmoid osteotomies with genioplasty, the root of the tree was the skull (ID1). Separating the neck from the skull, there were two nodes in the tree, the neck (ID2) and remaining part of the skull (ID3). Separating ID3 into ID6 and ID7, the maxilla (ID13) could be osteotomized from the skull without the mandible and the neck (ID6)-Le Fort I osteotomy. Four leaves with ID 26, 54, 110, and 111 were then assigned after the maxilla was segmentalized into four pieces. In the same principle, the mandible (ID7), also separated from ID3, was osteotomized as the right distal segment (ID14), the left distal segment (ID28), and the proximal segment (ID29) by bilateral subsigmoid osteotomies. Lastly, the chin segment (ID58) was osteotomized from proximal segment (ID29) by genioplasty. Fig. 9 shows a diagram of osteotomized bone segments with their unique ID.

After osteotomies, each bone segment with its own ID could be manipulated separately, including translation, rotation, eleva- 


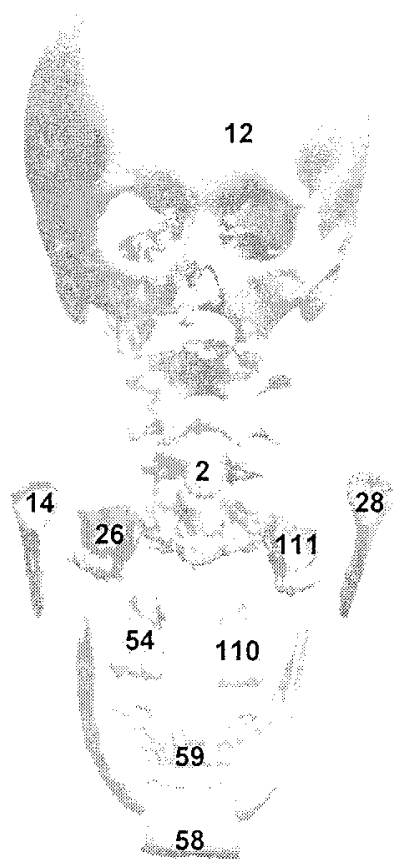

Fig. 9. Diagram of osteotomized bone segments.

tion, changing rotation center, displaying movement parameter, color masking, scaling, range boundaries, and switching each object to visible or invisible.

\section{3-D Soft-Tissue Planning and Prediction}

This was a soft-tissue deformation scheme for predicting the soft-tissue change before operation.

The movement of the osteotomized bone segment was assumed as a series of coordinate transformations in the 3-D space. After the bone segment was moved to a new position, the coordinates of feature points (vertices) on the bone segment were accordingly translated and rotated. The transformation matrices of bone segment were combined by matrix multiplication to achieve combinations of translation and rotation.

Two soft-tissue deformation algorithms were used in this scheme: the surface normal-based model deformation algorithm and the ray projection-based model deformation algorithm [10]. The corresponding relationship of the vertices of the triangles between the bone and soft-tissue surface was computed and saved as an intersection data file. The computation of the transformation of the soft-tissue model (individualized mesh) was then made by using a $4 \times 4$ transformation matrix. For the ray projection-based model deformation, the cylinder sampling model was applied to compute the intersection data along the ray projection from origin of coordinate, between eyebrow level and labiomental-fold level. The sphere sampling model was applied to calculate the remaining parts along the ray projection. If the surface normal of the vertex inferior to the plane of landmark labiomental fold was equal to or smaller than minus $10^{\circ}\left(\leq-10^{\circ}\right)$, the intersection data was computed along the direction of the surface normal instead of the ray projection for the surface normal-based model deformation.
To orchestrate the soft-tissue prediction with different operation types, the parameters of bone and soft-tissue movement ratios were transferred from intervened ratio files correspondingly [10].

3-D color facial texture-mapping technique was applied again to regenerate a color photorealistic facial model after soft-tissue deformation.

The soft-tissue changes were simply predicted by interactively and intraoperatively dragging the bone segments to the desired position. When the surgeon translated, rotated, and elevated each bone segment (Fig. 10), the soft-tissue model was correspondingly deformed and the texture was remapped from the cartograph in a real-time speed. A predicted 3-D facial visualization with color photorealistic quality was precisely created. Both the bone and soft-tissue models could be rotated freely and visualized immediately from arbitrary observing angle with zoom out or zoom in. Each object of the bone and soft tissue also could then be masked as visible or invisible in order to get the best visualization during the virtual operation. To check the soft-tissue and bone movement, the soft tissue could be displayed as a wire frame overlying on the bone. The predicted facial model could generate a sequence of images and be printed on glossy paper via a photo-quality color printer (Fig. 11).

\section{Clinical Trial}

To date, 14 cases have been planned using integrated 2-D and 3-D data and analysis. Surgical planning and simulation has been performed by this VRSP system. The postoperative visualization is predicted before operation.

The surgeon can use different combinations of shortcut keys, 2-D mouse, 3-D flying mouse, and CrystalEye eyewear, to perform the virtual osteotomies, to move bone segments, and simulate soft-tissue changes.

Based on the procedures and time requirements for analysis, six stages of treatment planning are available. The operator's clinical experience is important for achieving successful planning.

The first stage involves data acquisition and clinical evaluation, such as CT scanning, color-portrait capturing, cephalometric analysis, and primary clinical assessment. This requires a 2-7-h effort.

The second stage involves CT raw data post-processing, including removing undesired objects, enhancement processing, masking bone structure, etc. This requires a 2-3-h effort.

The third stage involves interactive visualization of the 3-D images, including the volumetric dataset generation, 3-D coordinates geometry transformation, etc. This requires about a 1-h effort.

The fourth stage of planning is to generate individual facial soft-tissue model with a color texture mapping. This is an important and semiautomatic step, which can directly affect the accuracy of predicted effects. This procedure requires about 1-2 $\mathrm{h}$.

The fifth stage of planning is to make decimation computation, to add digitizing landmark coordinates to the bone model in 

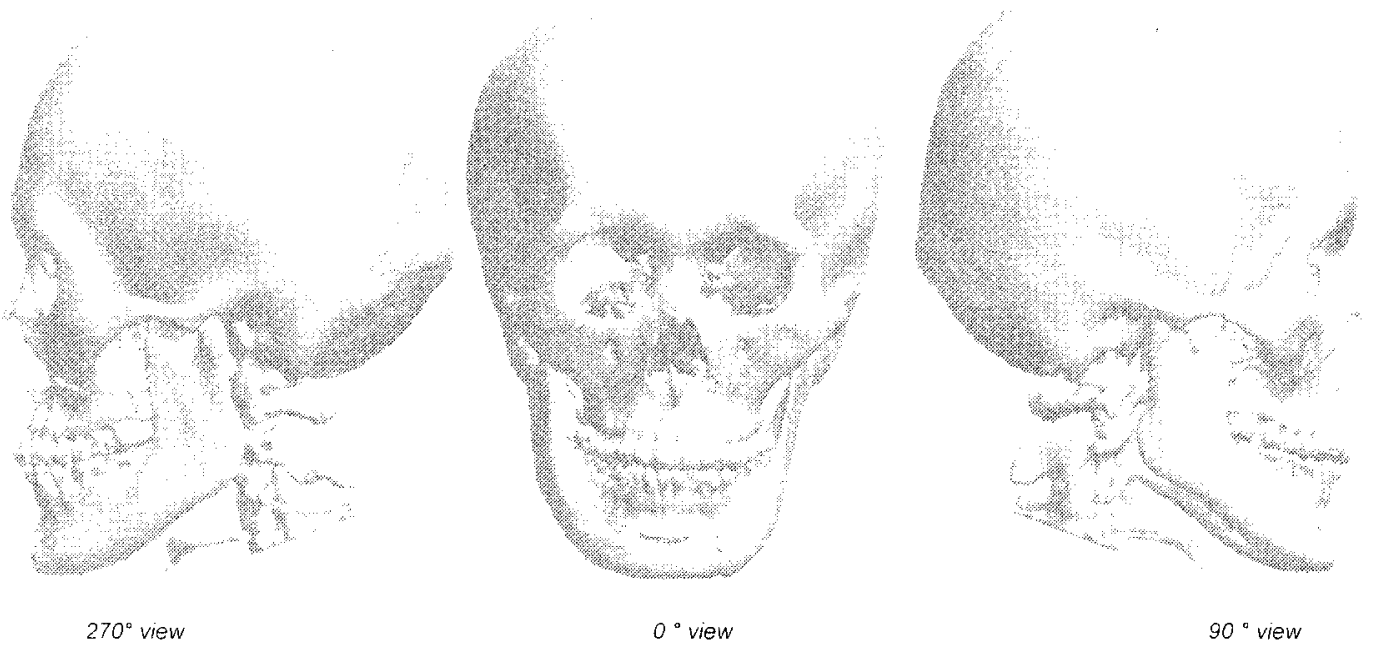

Fig. 10. Bone segments after movement.
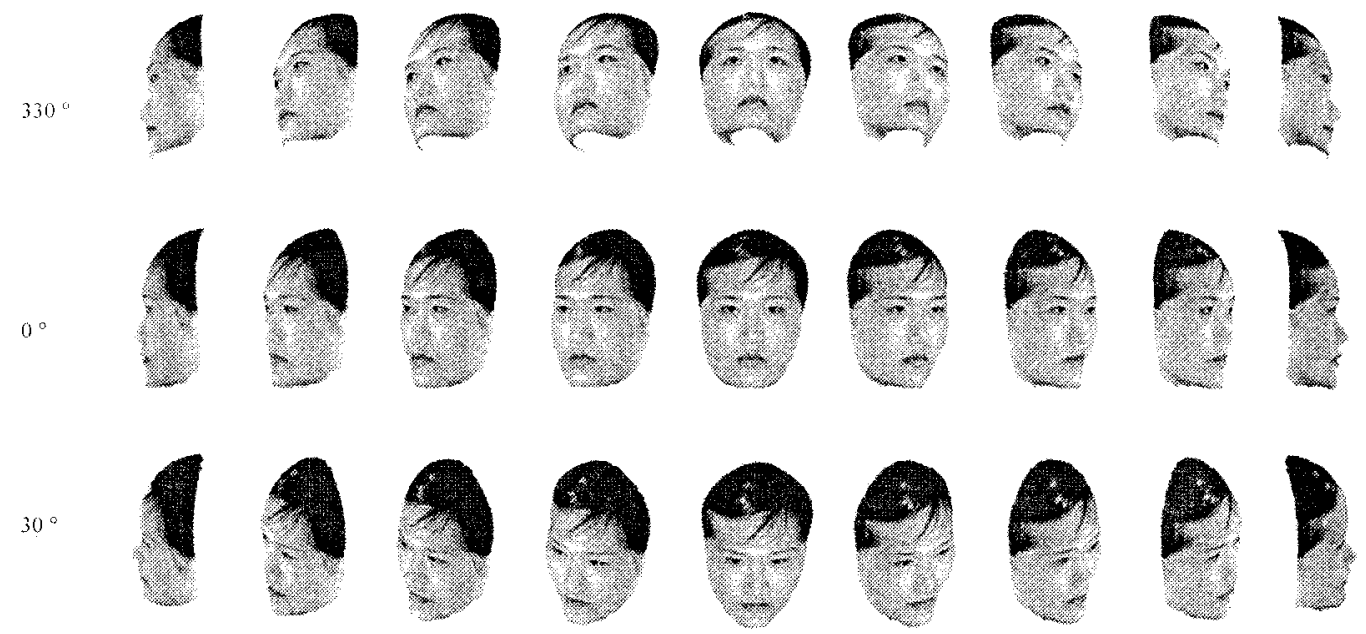

$270^{\circ}$

$300^{\circ}$

$315^{\circ}$

$330^{\circ}$

$0^{\circ}$

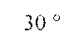

$45^{\circ}$

$60^{\circ}$

$90^{\circ}$

Fig. 11. Predicted image sequence.

order to perform varieties of procedures of virtual "osteotomies" in virtual-reality environment. This step is only operated on the bone. It requires about $1 \mathrm{~h}$ or less.

The sixth stage of planning is much quicker and requires about $10-15 \mathrm{~min}$. This step is totally operated in the virtual-reality environment with virtual-reality devices. The surgeon can move and rotate the osteotomized bone segments freely and observe the predicted result with full-color visualization. The operator may also compare results between the predicted and original facial models in 3-D visualization. If the predicted effectiveness is not satisfactory, the prediction can be discarded and repeated again. The movement data of a different bone segment is displayed on the computer monitor by selecting the required bone segment.

A planning and simulation session was held at the computer system with the maxillofacial team for review and final modifications. The quantitative translation and rotation data of each object could be displayed, respectively. The final result of planning and simulation can be visualized from arbitrary viewpoints in the virtual-reality workbench. It can also be produced as a sequence of color-texture remapped images and printed on glossy paper via any high-resolution photo-quality color printer (Fig. 11).

Follow-up was performed six months after operation. A comparison between original color portraits, predicted facial model, postoperative portraits, simulated osteotomies, and bone structures after surgery is shown in Figs. 12-14.

\section{DISCUSSION}

Surgical planning and simulation has wide applications, such as for orthognathic surgery, craniofacial surgery, cleft palate, trauma, distraction osteogenesis, etc. In general, surgical simulation is to perform the "surgery" in the computer and to con- 


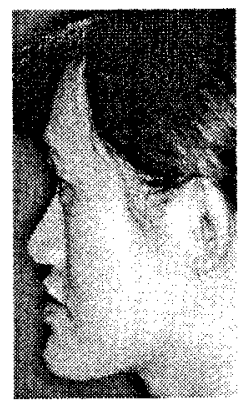

Before surgery

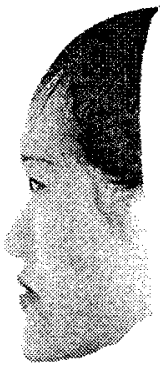

Simulated $3 D$ facial model

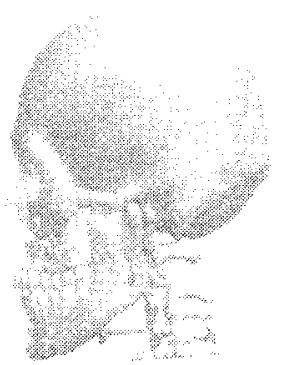

After osteotomies

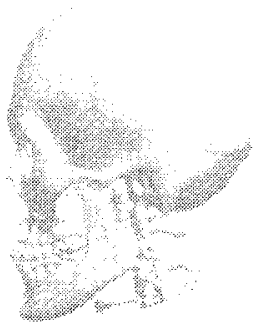

Simulated osteotomies

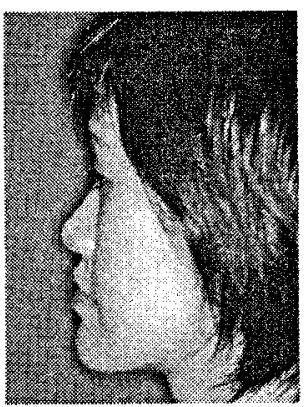

After surgery

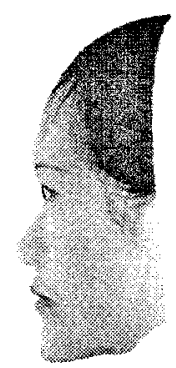

Predicted 3D facial model

Fig. 12. Comparison between predicted facial models and color portraits $\left(270^{\circ}\right.$ view $)$.

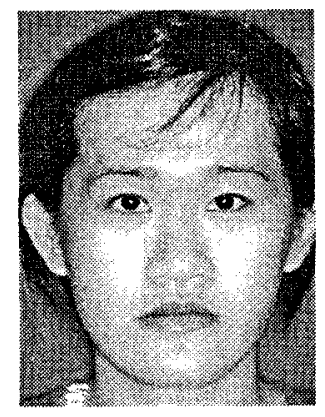

Before surgery

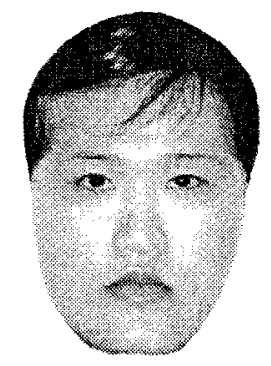

Simulated 30 facial model

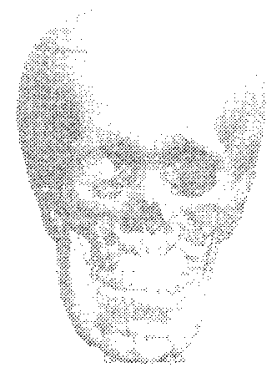

After osteotomies

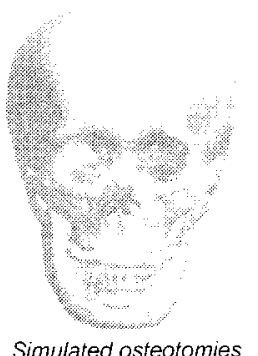

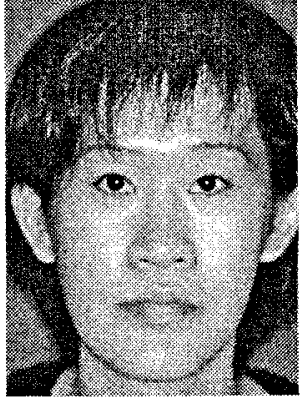

After surgery

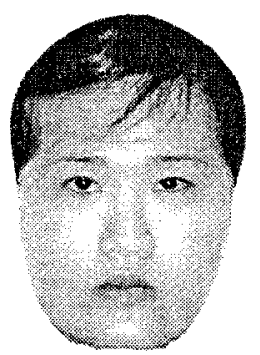

Predicted 3D facial model

Fig. 13. Comparison between predicted facial models and color portraits $\left(0^{\circ}\right.$ view $)$.

struct a "fake" image of the postsurgical appearance of the patient before operation.

3-D imaging technology is now widely available and used to aid in the comprehension and application of volumetric data to diagnosis, planning, and therapy [15]. A 3-D computer system and virtual-reality environment bring the surgeon into Cyberspace to perform the tasks that he performs routinely. $\mathrm{He}$ may immerse into the responsive workbench to gain the same feelings as if he is in the real situation. The computer itself no longer means everything in the whole system, but rather acts as a
"Daemon"- an intelligent server in the background, providing necessary information across multisensory interaction [9].

There are several studies on surgical planning for maxillofacial and craniofacial surgery [16]-[20]. Altobelli et al. [16] used the 3-D surface reconstruction from CT data to plan craniofacial operations. Cephalometric analysis was integrated with 3-D CT reconstruction to quantify the skeletal deformities and to assist in the design of the surgical procedure. Interactive techniques were developed to simulate osteotomies and skeletal movement in three dimensions on the computer-generated surface images. 


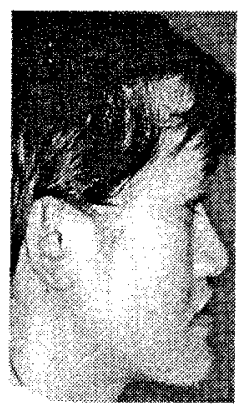

Before surgery

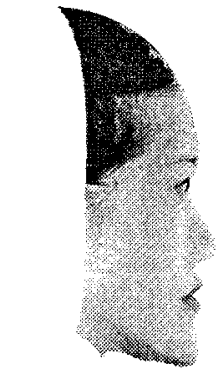

Simulated 3D facial model

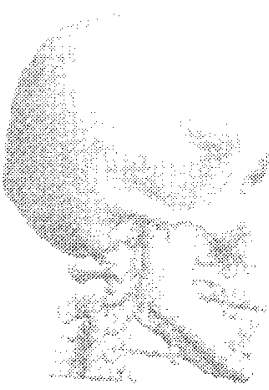

After osteotomies

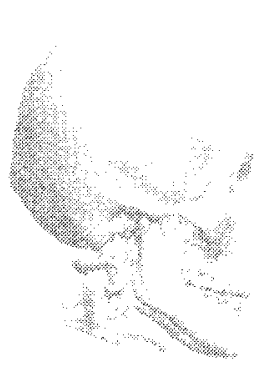

Simulated osteotomies

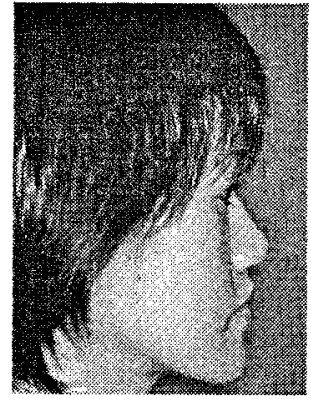

After surgery

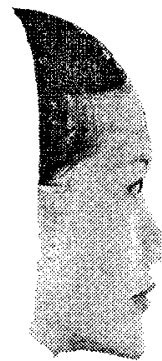

Predicted $3 D$ facial model

Fig. 14. Comparison between predicted facial models and color portraits $\left(90^{\circ}\right.$ view).

It took about one or two days for bone surgery simulation on an extremely powerful high-end graphic workstation. 3-D softtissue prediction based on bone movement was not included.

Stereolithographic biomodeling is a modern technology that allows 3-D CT data to be used to manufacture solid plastic replicas of anatomical structures (biomodels) [21]-[23]. It can produce the life-sized deformed bone structures, so a surgeon can directly observe a patient's deformity and make surgical planning on the biomodel before operation. However, it needs an extremely expensive machine to fabricate this biomodel, and soft-tissue-change prediction is still not included.

Soft-tissue-change prediction is an important aspect in surgical planning, especially in orthognathic surgery. The orthognathic surgery is not only to correct the malfunctions and to relocate malpositioned bones, but also for aesthetic purposes. Currently, soft-tissue simulation are still mainly focused on the 2-D profile prediction (color video-imaging techniques) combining with cephalometric analysis in two recent decades [24]-[28], only a few studies involved in three- dimensional soft-tissue capturing and deformation [3]-[7], [10], [29]-[32]. Ayoub et al. [29], [30] designed a vision-based 3-D facial data capture system (photogrammetry) for the planning of maxillofacial operations. The facial images and a cephalogram were captured simultaneously, followed by a computer program to create a 3-D facial model, which was used to predict soft-tissue changes of orthognathic surgery. However, the 2-D cephalometric radiographs may not have enough information to simulation 3-D soft-tissue changes. The relationship between soft tissue and bone still remains a question.

From the methods discussed above, there is no virtual-realitybased 3-D system to make precise orthognathic surgical planning and soft-tissue predictions with a PC. The aim of this study is to combine 3-D visualization, virtual reality, color video- imaging technique, surgical planning, and simulation in order to solve the bone-soft-tissue relationship problem and provide the surgeon with an ideal virtual-reality workbench for orthognathic surgical planning, simulation, and soft-tissue prediction in a personal computer system. The workbench is designed not only for surgical planning, but for teaching purposes as well. This study has achieved the following general objectives:

- to create a 3-D virtual-reality workbench for surgical planning, simulation, and soft-tissue prediction for orthognathic surgery;

- to establish a 3-D surface reconstruction from sequential CT data;

- to generate an individual 3-D model from a generic mesh;

- to perform color texture mapping from three views (frontal, right-hand, and left-hand side) of portraits;

- to generate fast 3-D visualization, so multiple virtual osteotomies can be real-time performed;

- to deform individual facial model (mesh) after virtual operation, so as to achieve soft-tissue prediction;

- to obtain a final product - the quantitative osteotomy-simulated 3-D bone model and the precise soft-tissue-change predicted 3-D facial model with color photorealistic quality, which can be real-time visualized in three dimensions and a virtual-reality environment;

- all procedures operated in a conventional personal computer system with relatively low-cost virtual-reality devices, instead of an extremely expensive high-end virtual-reality graphics workstation.

Considering the cost and radiation of whole-skull CT scanning to the patient, this workbench is more likely to be suitable for the cases with complex maxillofacial deformity, such as asymmetric deformity, cleft palate, distraction osteogenesis, and trauma, where radiation and the cost of a CT scan need to be justified. 


\section{ACKNOWLEDGMENT}

The authors would like to thank Dr. R. Kikinis, Surgical Planning Laboratory, Brigham and Women's Hospital, Harvard Medical School, Cambridge, MA, for his valuable suggestions and comments on this computer system. The authors also gratefully acknowledge F. Shen, Hong Kong Adventist Hospital, Hong Kong, for his expertise with CT scanning.

\section{REFERENCES}

[1] C. Ferencz and J. Greco, "A method for the three-dimensional study of pulmonary arteries," Chest, vol. 57, pp. 428-434, 1970.

[2] R. S. Pickering et al., "Computed tomography of the excised kidney," Radiology, vol. 113, pp. 643-647, 1974.

[3] A. M. McCance et al., "Three-dimensional analysis technique-Part 2: Laser scanning: A quantitative three-dimensional soft-tissue analysis using a color-coding system," Cleft Palate Craniofacial J., vol. 34, pp. 46-51, 1997.

[4] D. Terzopoulos and K. Waters, "Analysis and synthesis of facial image sequences using physical and anatomical models," IEEE Trans. Pattern, Anal. Machine Intell., vol. 15, pp. 569-579, June 1993.

[5] S. P. Studer et al., "Quantitative measurement of volume changes induced by oral plastic surgery: Validation of an optical method using different geometrically-formed specimens," J. Periodontics, vol. 68, pp. 950-962, 1997.

[6] K. Bush and O. Antonyshyn, "Three-dimensional facial anthropometry using a laser surface scanner: Validation of the technique," Plastic Reconstruct. Surgery, vol. 98, pp. 226-235, 1996.

[7] B. Lindqvist, U. Welander, and R. Mahler, "A three-dimensional photographic method for documentation and measurement of dental conditions," J. Orofacial Orthopedics, vol. 59, pp. 90-99, 1998.

[8] J. Xia et al., "Computer-assisted three-dimensional surgical planning and simulation-3D color facial model generation," Int. J. Oral Maxillofacial Surgery, vol. 29, pp. 2-10, 2000.

[9] J. Xia et al., "Computer-assisted three-dimensional surgical planning and simulation-3D virtual osteotomy," Int. J. Oral Maxillofacial Surgery, vol. 29, pp. 11-17, 2000.

[10] J. Xia et al., "Computer-assisted three- dimensional surgical planning and simulation-3D soft tissue planning and prediction," Int. J. Oral Maxillofacical Surgery, vol. 29, pp. 250-258, 2000.

[11] J. Xia et al., "Local feature based three- dimensional facial mapping from two side views," Computer-Aided Surgery, vol. 2, p. 211, 1997.

[12] H. E. Cline et al., "Two algorithms for the three-dimensional reconstruction of tomograms," Med. Phys., vol. 15, pp. 320-327, 1988.

[13] W. E. Lorensen and H. E. Cline, "Marching cubes: A high resolution 3D surface construction algorithm," Comput. Graph., vol. 21, pp. 163-169, 1987.

[14] W. Schroeder, J. Zarge, and W. Lorensen, "Decimation of triangle meshes," Comput. Graph., vol. 26, pp. 65-70, 1992.

[15] M. W. Vannier and J. L. Marsh, "Three-dimensional imaging, surgical planning, and image-guided therapy," Radiol. Clin. North Amer, vol. 34, pp. 545-563, 1996.

[16] D. E. Altobelli et al., "Computer-assisted three-dimensional planning in craniofacial surgery," Plastic Reconstruct. Surgery, vol. 92, pp. 576-585, 1993.

[17] F. R. Carls, B. Schuknecht, and H. F. Sailer, "Value of three-dimensional computed tomography in craniomaxillofacial surgery," J. Craniofacial Surgery, vol. 5, pp. 282-288, 1994.

[18] R. A. Fuhrmann, U. Frohberg, and N. Diedrich, "Treatment prediction with three-dimensional computer tomographic skull models," Amer. J. Orthodontic Dentofacial Orthopedics, vol. 106, pp. 156-160, 1994.

[19] A. M. McCance et al., "Three-dimensional analysis techniques-Part 1: Three-dimensional soft-tissue analysis of 24 adult cleft palate patients following Le Fort I maxillary advancement: A preliminary report," Cleft Palate Craniofacial J., vol. 34, pp. 36-45, 1997.

[20] M. W. Vannier, J. L. Marsh, and J. O. Warren, "Three-dimensional CT reconstruction images for craniofacial surgical planning and evaluation," Radiology, vol. 150, pp. 179-184, 1984.

[21] J. S. Bill et al., "Stereolithography in oral and maxillofacial operation planning," Int. J. Oral Maxillofacial Surgery, vol. 24, pp. 98-103, 1995.

[22] P. S. D’Urso et al., "Stereolithographic biomodeling in craniomaxillofacial surgery: A prospective trial," J. Craniomaxillofacial Surgery, vol. 27, pp. 30-37, 1999.
[23] D. M. Erickson et al., "An opinion survey of reported benefits from the use of stereolithographic models," J. Oral Maxillofacial Surgery, vol. 57, pp. 1041-1043, 1999.

[24] A. Gaggl, G. Schultes, and H. Karcher, "Changes in soft tissue profile after sagittal split ramus osteotomy and retropositioning of the mandible," J. Oral Maxillofacial Surgery, vol. 57, pp. 542-546, 1999.

[25] G. Schultes, A. Gaggl, and H. Karcher, "Accuracy of cephalometric and video imaging program dentofacial planner plus in orthognathic surgical planning," Computer-Aided Surgery, vol. 3, pp. 108-114, 1998.

[26] S. T. Syliangco et al., "Predicting soft tissue changes in mandibular advancement surgery: A comparison of two video imaging systems," Angle Orthodontists, vol. 67, pp. 337-346, 1997.

[27] J. Xia et al., "A study of computer aided simulation system of orthognathic surgery," Chin. J. Plastic Surgery Burns, vol. 10, pp. 278-281, 1994.

[28] J. Xia et al., "Computer aided simulation system of orthognathic surgery," in Proceedings of the Eighth IEEE Symposium on Computer-Based Medical Systems. Los Alamitos, CA: IEEE Comput. Soc. Press, 1995, pp. 237-244.

[29] A. F. Ayoub et al., "Three-dimensional modeling for modern diagnosis and planning in maxillofacial surgery," Int. J. Adult Orthodontic Orthognathic Surgery, vol. 11, pp. 225-233, 1996.

[30] A. F. Ayoub et al., "A vision-based three-dimensional capture system for maxillofacial assessment and surgical planning," Br. J. Oralmaxillofacial Surgery, vol. 36, pp. 353-357, 1998

[31] E. Keeve et al., "Deformable modeling of facial tissue for craniofacial surgery simulation," Computer-Aided Surgery, vol. 3, pp. 228-238, 1998.

[32] P. Techalertpaisarn and T. Kuroda, "Three-dimensional computer-graphic demonstration of facial soft tissue changes in mandibular prognathic patients after mandibular sagittal ramus osteotomy," Int. J. Adult Orthodontic Orthognathic Surgery, vol. 13, pp. 217-225, 1998.

James Xia (A'99-M'00), photograph and biography not available at time of publication.

Horace H. S. Ip (M'91), photograph and biography not available at time of publication.

Nabil Samman, photograph and biography not available at time of publication.

Helena T. F. Wong, photograph and biography not available at time of publication.

Jaime Gateno, photograph and biography not available at time of publication.

Dongfeng Wang, photograph and biography not available at time of publication.

Richie W. K. Yeung, photograph and biography not available at time of publication.

Christy S. B. Kot, photograph and biography not available at time of publication.

Henk Tideman, photograph and biography not available at time of publication. 